\title{
Infra-red techniques for thermomechanical characterisation of materials and structures
}

\author{
A. Chrysochoos • J. Dulieu-Barton
}

Published online: 21 March 2015

(C) Society for Experimental Mechanics 2015

The application of infra-red (IR) imaging techniques to the mechanics of materials and structures has grown considerably over past decades. The expansion is marked by the increased spatial and temporal resolution of the infra-red detectors, faster processing times and much greater temperature resolution. The improved sensitivity and more reliable temperature calibrations of the devices have meant that more accurate data can be obtained than were previously available. The purpose of the special issue is to bring together novel work on all aspects of thermomechanics with the focus on the application of IR imaging approaches. The main thrust is on the analysis of thermomechanical behavior of materials and using this behavior to elicit information on material characteristics, stress patterns, deformation mechanisms and failure modes. Of particular interest are strong thermomechanical couplings that result in complex, often nonlinear behaviour such as visco(thermos)elasticity, anisotropic heat diffusion mechanisms, strain and damage localisation and solid state phase changes. An objective is to share experience on how datarich experimental mechanics can help scientists and engineers to better understand and simulate the behavior of materials and structures. An important aspect is the use of other imaging techniques in conjunction with IR imaging, demonstrating how imaging is the route to a full thermomechanical characterization of both material behaviour and structural response.

The special issue starts with a paper that uses a technique known as thermoelastic stress analysis (TSA), which exploits

\footnotetext{
A. Chrysochoos

University of Montpellier, Montpellier, France

J. Dulieu-Barton $(\bowtie)$

University of Southampton, Southampton, UK

e-mail: janice@soton.ac.uk
}

the thermoelastic coupling that exists in materials under load. The thermoelastic coupling was first observed in the 1830s and the mathematical formulation of the thermoelastic effect was derived in the 1850s by Lord Kelvin. Essentially when a material experiences a strain change in the elastic range a small temperature change occurs that can be related to the trace of the stress tensor. In the 1980s single cell scanning IR detection systems were developed to obtain the 'thermoelastic response', essentially the output from system was a digital level. The first commercially available system was called SPATE 'Stress Pattern Analysis by Thermal Emissions', which was developed in the UK by and organization called SIRA 'Scientific Instruments Research Associations. The review by Lin, Saman, Khaja, and Rowlands, covers developments from the early days of SPATE to the present, where array based calibrated detection systems are used. One clear shortcoming of TSA is that can only deliver the sum of the principal stresses. The hybrid TSA approach described provides a route for determining the individual stress components by combining the measured thermal data with Airy stress functions.

In developing the formulation for TSA it is assumed that the small temperature change occurs isentropically. This is achieved by cyclically loading the specimen in the elastic range (reversible) and at such a rate that the thermal diffusion length is small (adiabatic). A key component of TSA is the use of a lock-in algorithm, which uses a reference signal (usually from a test machine signal generator), to process the data. The IR detection systems used for TSA are costly as they are cryogenically cooled photon detectors with fast framing rates that enable sufficient data to be captured to reconstruct the cyclic thermal response with great accuracy. The second paper in the issue is by Pitarresi and proposes an off-line lock-in correlation algorithm enabling TSA and lock-in thermography 
to be carried out using low cost single detector IR cameras with slow frame rates. The sampling and filtering strategies involve two alternative low pass filtering methods: one based on time averaging in the time domain and the other based on in the frequency domain by means of the fast Fourier transform (FFT). The work described in the paper offers new opportunities to apply TSA in structural health monitoring by the uses of multiple cameras.

The next two papers in the special issue are concerned with the thermal and dissipative effects associated with fatigue mechanisms. The first paper by Facchinetti, Florin, Doudard and Calloch concerns the self-heating observed during high cycle quasi-static fatigue tests (HCF) on a seam weld joint. Displacement and temperature fields are obtained using stereo Digital Image Correlation (DIC) and IR. A thermal source model is devised to provide a correlation between the change of the seam weld dissipative source and the expected mean fatigue strength of the assembly. The second paper by Blanche, Chrysochoos, Ranc, and Favier deals with energy dissipated during very high cycle fatigue tests (VHCF) at high loading frequency $(20 \mathrm{kHz})$ and low stress (i.e., far below the yield stress). The results obtained from tests on pure copper specimens show that dissipated energy exists whatever the attainable stress range and that the dissipated energy rate is not constant throughout the test. The important outcome is that the findings do not follow the concepts of fatigue limits based on elastic shakedown or the mechanical hysteresis loop (viscoplastic shakedown).

To link the mechanical and thermal variables it is possible to express the heat diffusion equation in terms of the heat sources induced by deformation mechanisms. The use of thermal data to estimate the distribution of heat sources is particularly challenging and requires a good knowledge of the thermo-physical properties of the materials and reliable temperature fields. The paper by Delobelle, Favier, Louche, and Connesson proposes an experimental protocol to estimate the heat capacity and thermal conductivity present in the heat diffusion equation, while the paper by Ranc, Blanche, Ryckelynck, Chrysochoos highlights the efficiency of proper orthogonal decomposition (POD) methods to remove noise from the thermal images before the heat source computation.

The next two papers in the issue use a combination of white light and IR imaging techniques to track the heterogeneous development of the microplasticity. Digital image correlation and point tracking of graphite dot markers are used to assess the strain fields. The paper by Charkaluk, Seghir, Bodelot, Witz, Dufrénoy focuses on the plastic localization at the grain scale and discusses the concept of yield stress at different observation scales. The paper by Oliferuk, Maj, Zembrzycki uses, at a more macroscopic scale, kinematic and thermal data to estimate the development of stored energy during strain localization to the evolution of the microplasticity.

The last group of papers in the special issue demonstrates the wide range of material behaviour that can be fruitfully studied using IR techniques. The paper by Chaiamarit, Balandraud, Preechawuttipong and Grédiac analyses a two dimensional cohesionless polydisperse granular materials under confined compression. IR thermography and TSA were used to analyse the hydrostatic stress network to estimate the force networks in granular media to check the consistency of discrete models based molecular dynamics. Another growing domain of IR applications is in studies of the behaviour of polymers and polymer composites. In the paper by Le Cam, Samaca Martinez, Balandraud, Toussaint, and Caillard the calorimetric effects accompanying the deformation of rubbers are studied. The temperature variations under deformation are measured using IR thermography. The heat sources produced or absorbed by the material are deduced from the temperature variations by using the heat diffusion equation. The calorimetric signatures of the most important effects in rubber deformation such as strain-induced crystallization and the Mullins effect, were characterised. Finally, the paper by El Yagoubi, Lamon, Batsale, Dhote, Le Flem deals with ceramic composites widely used in structural parts that are subjected to high temperatures or high temperature gradients. The anisotropic character of this kind of material leads the authors to develop special IR techniques to assess, at different scales, the anisotropic thermal diffusion properties of the composites.

The special issue on IR imaging and thermomechanics represents the state of the art in the topic and we believe marks the beginning of a much wider use of IR imaging to understand mechanics changing the emphasis from a tool for nondestructive evaluations. We thank all the authors of the papers for their painstaking efforts and we also thank the editorial board of Experimental Mechanics and the Society of Experimental Mechanics for inviting us to produce the special issue. 